\title{
Early markers of obesity-related renal injury in childhood
}

\author{
Wei Ding $\cdot$ Robert H Mak
}

Received: 23 September 2014 / Revised: 24 September 2014 / Accepted: 26 September 2014 / Published online: 17 October 2014

(C) IPNA 2014

\begin{abstract}
Childhood obesity has become a global epidemic. Recent epidemiological data suggest that obesity is associated with increased risk of renal injury in children. The onset of obesity-associated renal disease is insidious and asymptomatic, so early markers will be extremely useful in its prevention and treatment. Biomarker discovery can be focused on unbiased or biased (candidate) approaches. Unbiased approaches using innovative technologies, such as proteomics and metabolomics, have uncovered candidates that are emerging as plausible biomarkers for such renal disorders as obstructive uropathy and diabetic nephropathy. Biased approaches are based on hypotheses related to glomerular or tubular injury pathophysiology in obesity. Goknar et al. (Pediatric Nephrology 2014; doi: 10.1007/s00467-014-2829-0) recently evaluated early urine renal injury markers, namely, microalbuminuria, $\mathrm{N}$-acetyl-beta-D-glucosaminidase (NAG), neutrophil gelatinase-associated lipocalin, and kidney injury molecule (KIM)-1, in obese children. They reported that obese children had higher urinary NAG and KIM-1 levels than healthy controls. Longitudinal observation studies are needed to evaluate whether these tubular damage markers are useful as early markers of renal injury in obese children.
\end{abstract}

Keywords Biomarkers · Obesity · Early urine markers .

Renal injury $\cdot$ Microalbuminuria .

$\mathrm{N}$-acetyl-beta-D-glucosaminidase $\cdot$ Kidney injury molecule-1

W. Ding $\cdot$ R. H. Mak $(\bowtie)$

Division of Pediatric Nephrology, University of California, 9500

Gilman Drive, Mailcode 0634, La Jolla, CA 92093, USA

e-mail: romak@ucsd.edu

W. Ding

Division of Nephrology, 5th People's Hospital of Shanghai, Fudan University, Shanghai, China

\section{Introduction}

Childhood obesity is fast becoming a worldwide epidemic. Recent epidemiological data suggest that obesity is associated with increased risk of renal injury in children [1]. Hsu et al. demonstrated the effect of body mass index (BMI) on the risk of end-stage renal disease in a cohort of 330,252 persons in California between 1964 and 1985, reporting a strong doseresponse relationship between the baseline BMI and the risk of chronic kidney disease (CKD) [2]. Filler et al. demonstrated that pediatric renal patients had significantly higher BMI $z$ scores than the normal population at a tertiary center in Canada over a period of 2 decades [3]. In children with renal transplants, kidney samples obtained from obese donors (BMI $>30$ ) had a lower glomerular filtration rate (GFR) and higher allograft dysfunction rate than kidney samples obtained from lean individuals $(\mathrm{BMI}<25)[4]$. These recent studies suggest that obesity is an independent risk factor for CKD in children. The onset of obesity-associated renal disease is insidious and asymptomatic, so early markers will be extremely useful in its prevention and treatment.

\section{Biomarkers of renal injury}

A biomarker is defined as a characteristic that can be objectively measured and evaluated as an indicator of normal biological or pathogenic processes or pharmacological responses to a therapeutic intervention. Biomarker discovery can be focused on unbiased or biased (candidate) approaches.

Unbiased approaches using such innovative technologies as proteomics and metabolomics have uncovered candidates that are emerging as plausible biomarkers for kidney diseases. Decramer et al. identified polypeptides that enabled diagnosis of the severity of obstruction and validated these biomarkers in urine collected in a prospective blind study. Using these 
noninvasive biomarkers, these authors were able to predict, several months in advance and with $94 \%$ precision, the clinical evolution of neonates with ureteropelvic junction obstruction [5]. Sharma et al. used metabolomics approaches to quantify 94 urine metabolites in screening and validation cohorts of patients with diabetes mellitus (DM) and CKD $(\mathrm{DM}+\mathrm{CKD})$, in patients with DM without CKD (DM $\mathrm{CKD}$ ), and in healthy controls [6]. Comparison of all these metabolites with the levels in healthy controls revealed that 13 metabolites were significantly reduced in the $(\mathrm{DM}+\mathrm{CKD})$ cohorts $(P \leq 0.001)$ and that 12 of these 13 remained significant when compared with the (DM - CKD) cohort. Analysis of the bioinformatics data indicated that 12 of the 13 differentially expressed metabolites are linked to mitochondrial metabolism, suggesting global suppression of mitochondrial activity in diabetic kidney disease [6].

Biased approaches are based on hypotheses related to glomerular or tubular injury pathophysiology. Examples of biomarkers for renal injury are proteins, lipids, microRNAs, genomic, metabolomic, or proteomic patterns, imaging determinations, electrical signals, and cells present on a urinalysis. A partial list of candidate markers for kidney injury is present in Table 1 with the corresponding sites along the nephron [7]. Legacy kidney biomarkers include serum creatinine $(\mathrm{sCr})$, blood urea nitrogen $(\mathrm{BUN})$, urinary albumin/protein, and volume excretion. However, neither sCr nor BUN can distinguish injury from hemodynamic changes in the kidney that

Table 1 Candidate biomarkers for renal injury

\begin{tabular}{llll}
\hline $\begin{array}{l}\text { Glomerular } \\
\text { injury markers }\end{array}$ & $\begin{array}{l}\text { Proximal tubules } \\
\text { injury markers }\end{array}$ & $\begin{array}{l}\text { Distal tubules } \\
\text { injury markers }\end{array}$ & $\begin{array}{l}\text { Collecting duct } \\
\text { injury markers }\end{array}$ \\
\hline $\begin{array}{l}\text { Albumin } \\
\text { Creatinine }\end{array}$ & $\begin{array}{l}\text { Cystatin C } \\
\text { Exosomal } \\
\text { fetuin-A }\end{array}$ & $\begin{array}{l}\text { Calbindin D28 } \\
\text { Clusterin }\end{array}$ & Calbindin D28 \\
Cystatin C & L-FABP & GST- $\mu / \pi$ & \\
Podocalyxin & GST-a & H-FABP & \\
$\beta 2-$ & microglobulin & HGF, NHE3 & IL-18 \\
& & Lipocalin & \\
WT-1 & IL-18 & (NGAL) & \\
& Kim-1 & NAG & \\
& Lipocalin & & \\
& (NGAL) & & \\
& NAG & & \\
& Netrin-1 & \\
& Osteopontin &
\end{tabular}

WT-1, Wilms tumor protein-1; FABP, fatty acid binding protein; GST, glutathione- $S$-transferase; HGF, hepatocyte growth factor; NHE3, sodium-hydrogen exchanger 3; IL, interleukin; NGAL, Kim-1, kidney injury molecule 1; NGAL, neutrophil gelatinase-associated lipocalin; NAG, $N$ acetyl-beta-D-glucosaminidase lead to appropriate changes in the GFR, particularly when the changes are acute. Furthermore, neither $\mathrm{sCr}$ nor BUN can change quickly enough with injury since individuals with normal renal function have a functional reserve that is brought into play in response to nephron injury. This reserve consists of other nephrons increasing their function so that $\mathrm{sCr}$ and BUN may not move out of the "normal range" until there is a great deal of injury and potentially irreversible loss of nephrons. Thus, GFR, whether measured by sCr or by more direct methods such as iohexol clearance, is a measure of kidney function, which is clearly important but which may not move in synchrony with injury. Having a biomarker that directly reflects injury and which is easily measured from a body fluid that is easily obtained, such as blood or urine, would change the paradigm to facilitate direct monitoring of injury rather than a secondary consequence of injury, such as a delayed reduction in GFR. Multiple markers will be useful in nephrology as they will provide different information; for example, about site of injury, involvement of inflammation, and system associations. Some characteristics of an ideal biomarker are presented in Table 2. Biomarkers can be classified as predictive, prognostic, diagnostic, or pharmacodynamic markers, or as efficacy or surrogate markers [8].

\section{Biomarker qualification and validation}

Most researchers agree that a new generation of predictive biomarkers would dramatically improve the efficiency of product development, help identify safety problems before a product is on the market (even before it is tested in humans), and facilitate the development of new types of clinical trials that will produce better data faster. The U.S. Food and Drug Administration (FDA) has developed a very thoughtful process by which they will "qualify" biomarkers in a "fit for purpose" contextualized way [9]. There is a fundamental difference between "qualification" and "validation" of a biomarker. The latter term is best used to connote the validity and harmonization across many laboratories of the assay used to quantitate the biomarker. The FDA has provided guidance that a biomarker can be considered "valid" if (1) it is measured in an analytical test system with well-established performance characteristics, and (2) there is an established scientific framework or body of evidence that elucidates the physiological, pharmacological, toxicological, and/or clinical significance of the test result [10].

\section{Biomarkers of early renal injury in obese children}

Goknar et al. recently evaluated early urine renal injury markers in obese children [11]. In this section we discuss their 
Table 2 Characteristics of an ideal biomarker

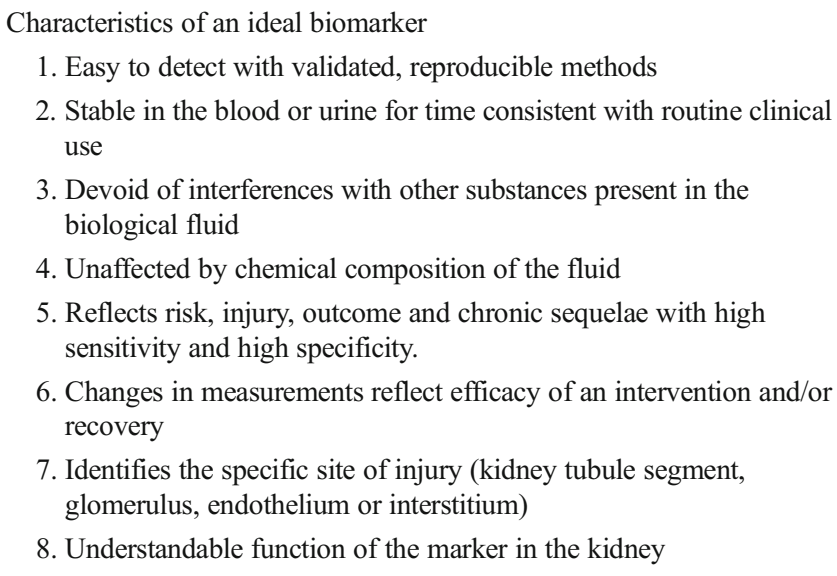

findings and review other published evidence on the validity of these markers.

\section{Microalbuminuria}

Microalbuminuria, an early marker of CKD, is also considered to be an early marker of renal injury in nondiabetic patients. This condition is quite common in obese children and adolescents. Csernus et al. showed elevated levels of albuminuria and $\beta 2$-microglobulinuria in obese children versus normal weight children, suggesting early renal glomerular and tubular injury as a consequence of obesity in children [12]. Ferris et al. demonstrated that microalbuminuria was strongly related to the severity of obesity in adults. In addition, these authors reported that a change in BMI over the 6 years of the study, but not the baseline BMI itself, was associated with microalbuminuria, indicating that the growth in body mass may occur faster than the kidneys can adapt [13]. Microalbuminuria was not found to be different between obese children and healthy controls in the study of Goknar et al. [11].

Neutrophil gelatinase-associated lipocalin

Neutrophil gelatinase-associated lipocalin (NGAL), a 25-kDa protein, is known to be released from injured renal tubular cells in acute kidney injury (AKI) before a decrease in the GFR can be detected [14]. In patients with moderate kidney disease, serum and urine NGAL concentrations have been identified to be independent predictors of CKD progression [15]. Furthermore, urine NGAL can be used as an early biomarker of diabetic nephropathy [16]. However, urine NGAL was not found to be different between obese children and healthy controls in Goknar et al.'s study [11].
$\boldsymbol{N}$-acetyl-beta-D-glucosaminidase

$N$-acetyl-beta-D-glucosaminidase (NAG), a lysosomal enzyme found predominantly in proximal tubules, is normally excreted in low amounts as a consequence of the physiological exocytosis process. Therefore, increased expression of NAG suggests injury to tubular cells and could be recognized as an early urinary marker for tubular cell malfunction. Several lines of evidence support the notion that NAG may be an early reliable marker of obesity-related renal injury. Mousa and co-workers demonstrated that the level of urinary NAG excretion was significantly increased in hypertensive patients compared with normotensive subjects. The upregulated urinary NAG excretion statistically correlated with patient age, serum uric acid, and duration of hypertension [17]. Zhang et al. tracked the progression of nephropathy in hypertensive and obese SHROB rats versus control hypertensive SHR-lean rats. Their results indicate that urinary NAG was elevated at week 33 and 36 in SHROB rats only, but not in SHR-lean rats at any time point [18], indicating that NAG may be an early reliable marker for obesity-related renal injury. Goknar et al. demonstrated that urinary NAG was significantly elevated in obese children compared with lean controls [11].

\section{Kidney injury molecule}

Kidney injury molecule (KIM-1) is a transmembrane glycoprotein, and its protein expression is not detectable in the normal kidney. The soluble form of KIM-1 can be easily detected in the urine of patients with AKI and can be used as an important biomarker for tubular injury [19]. In a casecontrol study of 40 children undergoing cardiac surgery, including 20 children with AKI (defined as a $50 \%$ increase in $\mathrm{SCr}$ ) and 20 without AKI, urinary KIM-1 levels were significantly increased, with an area under the time-concentration curve of 0.83 at the 12-h time-point [20]. Goknar et al.'s study showed that obese children had a higher urinary KIM-1 level compared with healthy controls [11]. These results suggest that KIM-1 may be a potential screening biomarker for the detection of early renal injury in obese children.

\section{Conclusion}

The link between obesity and CKD is of enormous public health importance. There is convincing evidence to suggest that overweight and obesity are significantly associated with increased risk of renal injury in adults and possibly also in children. Goknar et al. evaluated early urine renal injury markers, namely microalbuminuria, NAG, NGAL, and KIM-1 in obese children and reported that obese children had higher urinary NAG and KIM-1 levels compared with 
healthy controls [11]. They also found that urinary NAG and KIM-1 were not significantly different in obese children when comorbidities such as impaired glucose tolerance, insulin resistance, and hypertension were considered. Although a formal multivariant analysis was not performed, these findings indicate that these early renal injury markers may be specific for obesity-associated renal injury. Longitudinal observation studies are needed to evaluate whether these tubular damage markers are useful as early renal injury markers for obese children, in keeping with the FDA guidelines for qualification and validation.

Acknowledgments RHK is supported by National Institute of Health U01 DK-3-012 and R24HD050837 grants. WD is supported by a fellowship grant from Fudan University, China.

\section{References}

1. Gunta SS, Mak RH (2013) Is obesity a risk factor for chronic kidney disease in children? Pediatr Nephrol 28:1949-1956

2. Hsu CY, McCulloch CE, Iribarren C, Darbinian J, Go AS (2006) Body mass index and risk for end-stage renal disease. Ann Intern Med 144:21-28

3. Filler G, Reimao SM, Kathiravelu A, Grimmer J, Feber J, Drukker A (2007) Pediatric nephrology patients are overweight: 20 years' experience in a single Canadian tertiary pediatric nephrology clinic. Int Urol Nephrol 39:1235-1240

4. Espinoza R, Gracida C, Cancino J, Ibarra A (2006) Effect of obese living donors on the outcome and metabolic features in recipients of kidney transplantation. Transplant Proc 38:888-889

5. Decramer S, Wittke S, Mischak H, Zurbig P, Walden M, Bouissou F, Bascands JL, Schanstra JP (2006) Predicting the clinical outcome of congenital unilateral ureteropelvic junction obstruction in newborn by urinary proteome analysis. Nat Med 12:398-400

6. Sharma K, Karl B, Mathew AV, Gangoiti JA, Wassel CL, Saito R, Pu M, Sharma S, You YH, Wang L, Diomond-Stanic M, Lindenmeyer MT, Forsblom C, Wu W, Ix JH, Ideker T, Kopp JB, Nigam SK, Cohen CD, Groop PH, Barshop BA, Natarajan L, Nyhan WL, Naviaux RK (2013) Metabolomics reveals signature of mitochondrial dysfunction in diabetic kidney disease. J Am Soc Nephrol 24: 1901-1912
7. Bonventre JV, Vaidya VS, Schmouder R, Feig P, Dieterle F (2010) Next-generation biomarkers for detecting kidney toxicity. Nat Biotechnol 28:436-440

8. Bonventre JV (2014) Current biomarkers in kidney disease: dawning of a new era. ASN Kidney News 7-8

9. Goodsaid F, Papaluca (2010) Evolution of biomarker qualification at the health authorities. Nat Biotechnol 28:441-443

10. U.S. Food and Drug Administration (FDA) (2005) Guidance for industry. U.S. FDA, Silver Spring

11. Goknar N, Oktem F, Ozgen IT, Torun E, Kucukkoc M, Demir AD, Cesur Y (2014) Determination of early urinary renal injury markers in obese children. Pediatr Nephrol. doi:10.1007/s00467-014-2829-0

12. Csernus K, Lanyi E, Erhardt E, Molnar D (2005) Effect of childhood obesity and obesity-related cardiovascular risk factors on glomerular and tubular protein excretion. Eur J Pediatr 164:44-49

13. Ferris M, Hogan SL, Chin H, Shoham DA, Gipson DS, Gibson K, Yilmaz S, Falk RJ, Jennette JC (2007) Obesity, albuminuria, and urinalysis findings in US young adults from the Add Health Wave III study. Clin J Am Soc Nephrol 2:1207-1214

14. Mishra J, Ma Q, Prada A, Mitsnefes M, Zahedi K, Yang J, Barasch J, Devarajan P (2003) Identification of neutrophil gelatinase-associated lipocalin as a novel early urinary biomarker for ischemic renal injury. J Am Soc Nephrol 14:2534-43

15. Bolignano D, Lacquaniti A, Coppolino G, Donato V, Campo S, Fazio MR, Nicocia G, Buemi M (2009) Neutrophil gelatinase-associated lipocalin (NGAL) and progression of chronic kidney disease. Clin $\mathrm{J}$ Am Soc Nephrol 4:337-344

16. Bolignano D, Lacquaniti A, Coppolino G, Donato V, Fazio MR, Nicocia G, Buemi M (2009) Neutrophil gelatinase-associated lipocalin as an early biomarker of nephropathy in diabetic patients. Kidney Blood Press Res 32:91-98

17. Mousa AA, EI-Eshmawy MM, El-Adawy EH, Arafa LF, Badrawy AE, Saleh OM (2012) Renal resistive index and urinary $\mathrm{N}$-acetyl- $\beta$-glucosaminidase as predictors of early renal involvement in patients with essential hypertension. World J Nephrol Urol 1:36-41

18. Zhang Q, Davis KJ, Hoffmann D, Vaidya VS, Brown RP, Goering PL (2014) Urinary biomarkers track the progression of nephropathy in hypertensive and obese rats. Biomark Med 8:85-94

19. Han WK, Bailly V, Abichandani R, Thadhani R, Bonventre JV (2002) Kidney Injury Molecule-1 (KIM-1): a novel biomarker for human renal proximal tubule injury. Kidney Int 62: 237-244

20. Han WK, Waikar SS, Johnson A, Betensky RA, Dent CL, Devarajan P, Bonventre JV (2008) Urinary biomarkers in the early diagnosis of acute kidney injury. Kidney Int 73:863-869 